\title{
TNFAIP3 mutation may be associated with favorable overall survival for patients with T-cell lymphoma
}

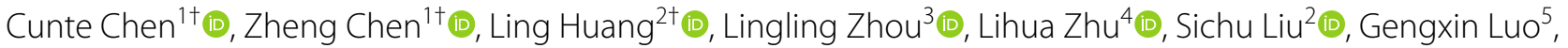

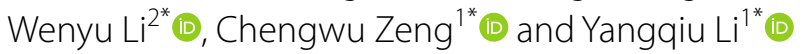

\begin{abstract}
Background: T-cell lymphoma (TCL) is highly aggressive and has a poor prognosis; thus, it is worth exploring biomarkers that may predict clinical outcomes and investigate their potential role in developing targeted therapies. In this study, we characterized the mutation pattern of tumor necrosis factor-alpha-inducing protein 3 (TNFAIP3) and its role in the prognosis of TCL patients.

Methods: Coding sequence (CDS) mutations in TNFAIP3 in TCL patients was explored using exome-sequencing data from 79 patients in our center (Guangdong Provincial People's Hospital, GDPH) and 544 samples from the Catalogue of Somatic Mutations in Cancer (COSMIC) database. Additionally, non-CDS mutations in TNFAIP3 in 41 TCL patients from our center (JNU) were investigated by polymerase chain reaction (PCR) and Sanger sequencing. Furthermore, non-CDS mutations in TNFAIP3 in $47 \mathrm{TCL}$ patients from Gene Expression Omnibus (GEO) dataset were explored.

Results: In the COSMIC database, TNFAIP3 mutations in TCL patients were located in the CDS, and the overall mutation frequency was $2.2 \%$. However, TNFAIP3 mutations were not detected in the CDS of any of the samples in our center's datasets. Interestingly, non-CDS TNFAIP3 mutations were found in $14.6 \%$ and $4.3 \%$ of TCL patients in the JNU and GSE15842 dataset, respectively. Importantly, there was a clear trend showing that TCL patients with a TNFAIP3 mutation were associated with a longer 5-year restricted mean survival time (RMST) and favorable OS rate compared with those without a TNFAIP3 mutation in the JNU dataset [hazard ratio $(\mathrm{HR})=0.29,95 \%$ confidence interval (Cl) 0.07 to $1.31, P=0.089]$. Furthermore, TNFAIP3 mutations significantly correlated with T-cell large granular lymphocytic leukemia (T-LGLL) with a favorable prognosis in the JNU dataset $(P=0.002)$. Notably, the different mutation patterns of TNFAIP3 when comparing our center and the COSMIC datasets might be due to different ethnic and genetic backgrounds.
\end{abstract}

\footnotetext{
*Correspondence: liwenyu@gdph.org.cn; zengcw@jnu.edu.cn;

tyangqiuli@jnu.edu.cn; yangqiuli@hotmail.com

${ }^{\dagger}$ Cunte Chen, Zheng Chen and Ling Huang contributed equally to this

work

${ }^{1}$ Institute of Hematology, School of Medicine, Key Laboratory

for Regenerative Medicine of Ministry of Education, Jinan University,

Guangzhou 510632, China

${ }^{2}$ Department of Lymphoma, Guangdong Provincial People's Hospital,

Guangdong Academy of Medical Sciences, School of Medicine, South

China University of Technology, Guangzhou, China

Full list of author information is available at the end of the article
}

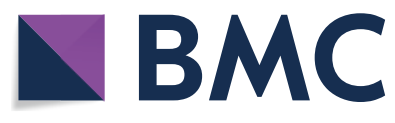

2021. Open Access This article is licensed under a Creative Commons Attribution 4.0 International License, which permits use, sharing, adaptation, distribution and reproduction in any medium or format, as long as you give appropriate credit to the original author(s) and the source, provide a link to the Creative Commons licence, and indicate if changes were made. The images or other third party material in this article are included in the article's Creative Commons licence, unless indicated otherwise in a credit line to the material. If material is not included in the article's Creative Commons licence and your intended use is not permitted by statutory regulation or exceeds the permitted use, you will need to obtain permission directly from the copyright holder. To view a copy of this licence, visit http://creativecommons.org/licenses/by/4.0/. The Creative Commons Public Domain Dedication waiver (http://creativeco mmons.org/publicdomain/zero/1.0/) applies to the data made available in this article, unless otherwise stated in a credit line to the data. 
Conclusions: To the best of our knowledge, we for the first time describe that TNFAIP3 mutations in non-CDS regions are associated with favorable OS for TCL patients, which might be a potential biomarker for the prognostic stratification of Chinese TCL patients.

Keywords: TNFAIP3 mutation, Mutation pattern, T-cell lymphoma, Prognosis

\section{Introduction}

T-cell lymphoma (TCL) originates from lymphoblasts or mature $\mathrm{T}$ cells. This disease accounts for only $10-15 \%$ of non-Hodgkin's lymphoma and can be further subdivided into many relatively rare subtypes [1-5]. Patients with TCL tend to have a poor prognosis when compared to those with B-cell lymphoma [6-8]. Implementation of a risk stratification model based on the international prognosis index (IPI) has made significant progress in predicting the prognosis of TCL patients, and this refined stratification can influence clinical decision-making, thereby improving the prognosis of patients $[9,10]$. However, the current risk stratification based on IPI cannot accurately predict prognoses for all TCL patients $[9,11]$. Therefore, it is worth exploring novel biomarkers that further improve risk stratification for TCL.

The intracellular ubiquitin editing protein tumor necrosis factor alpha-induced protein 3 (TNFAIP3), also known as A20, negatively regulates the activity of NF- $\mathrm{KB}$ in a variety of pathways through tumor necrosis factor and Toll-like receptors [12-14]. The TNFAIP3 gene locus is located in chromosome band $6 q 23$, and its deletion frequently occurs in B-cell lymphomas, particularly extranodal marginal zone $B$ cell lymphoma and diffuse large B-cell lymphoma [15-17]. Additionally, a few previous studies have revealed that TNFAIP3 deletion is frequently found in cutaneous T-cell lymphoma (CTCL) and NK-T cell lymphoma (NKTCL) $[15,18,19]$. Moreover, the prognostic value of TNFAIP3 deletion in NKTCL patients has been investigated, but the results are contradictory $[15,19]$. In our previous study, mutations in the non-coding sequence (non-CDS) region of TNFAIP3 were also found in T-cell hematological malignancies $[20,21]$. However, comprehensive assessment of the mutation pattern and prognostic importance of TNFAIP3 in Chinese TCL patients in multiple large datasets remains lacking.

In this study, we investigated the TNFAIP3 mutation pattern and its prognostic value for TCL patients from Jinan University (JNU), Guangdong Provincial People's Hospital (GDPH), Catalogue of Somatic Mutations in Cancer (COSMIC), and Gene Expression Omnibus (GEO) datasets.

\section{Materials and methods GDPH and JNU datasets}

A total of 79 tumor biopsies and whole blood samples were collected from de novo TCL patients at GDPH between February 27, 2015 and December 17, 2020, and these samples were used for exome sequencing. In the GDPH dataset, the TCL subtypes accounting for greater than $10 \%$ of the samples were as follows: T-lymphoblastic lymphoma (T-LBL) (22.8\%), angioimmunoblastic T-cell lymphoma (AITL) (22.8\%), peripheral T-cell lymphoma, not otherwise specified (PTCL-NOS) (22.8\%), and NKTCL (19.0\%). Moreover, peripheral blood mononuclear cells (PBMCs) from 41 de novo TCL patients from JNU between October 19, 2007 and January 27, 2016 and 30 healthy individuals (HIs) were obtained for PCR and Sanger sequencing $[20,21]$ (Fig. 1). In the JNU dataset, TCL subtypes accounting for greater than $10 \%$ of the samples were as follows: NKTCL (36.6\%), T-LBL (22.0\%), AITL (12.2\%), and PTCL-NOS (12.2\%). Corresponding clinical information, including gender, age, status, and overall survival (OS) time, was also collected (Table 1). The patients in the JNU and GDPH datasets are all from China (100\%). TCL subtypes were determined according to the 2008 World Health Organization (WHO) classification of tumors of hematopoietic and lymphoid tissues [22]. The date of the last follow-up was May 1, 2021, and the median follow-up time for the surviving TCL patients in the JNU dataset was 23.37 months (range: $0.23-157.83$ months). OS was defined as the span from diagnosis to death from any cause or last follow-up. All procedures involving human participants were performed according to the principles of the Declaration of Helsinki and approved by the Ethics Committee of the First Affiliated Hospital of Jinan University. All patients provided written informed consent.

\section{COSMIC and GSE15842 datasets}

Mutation data from 544 TCL patients from 12 clinical centers were downloaded from the COSMIC database (https://cancer.sanger.ac.uk/cosmic). Furthermore, single nucleotide polymorphism (SNP) data of 47 German patients with PTCL-NOS in GSE15842 datasets were downloaded from the GEO database (https://www.ncbi. nlm.nih.gov/geo/). In addition, the countries of origin for the TCL patients as well as TCL subtypes were obtained 


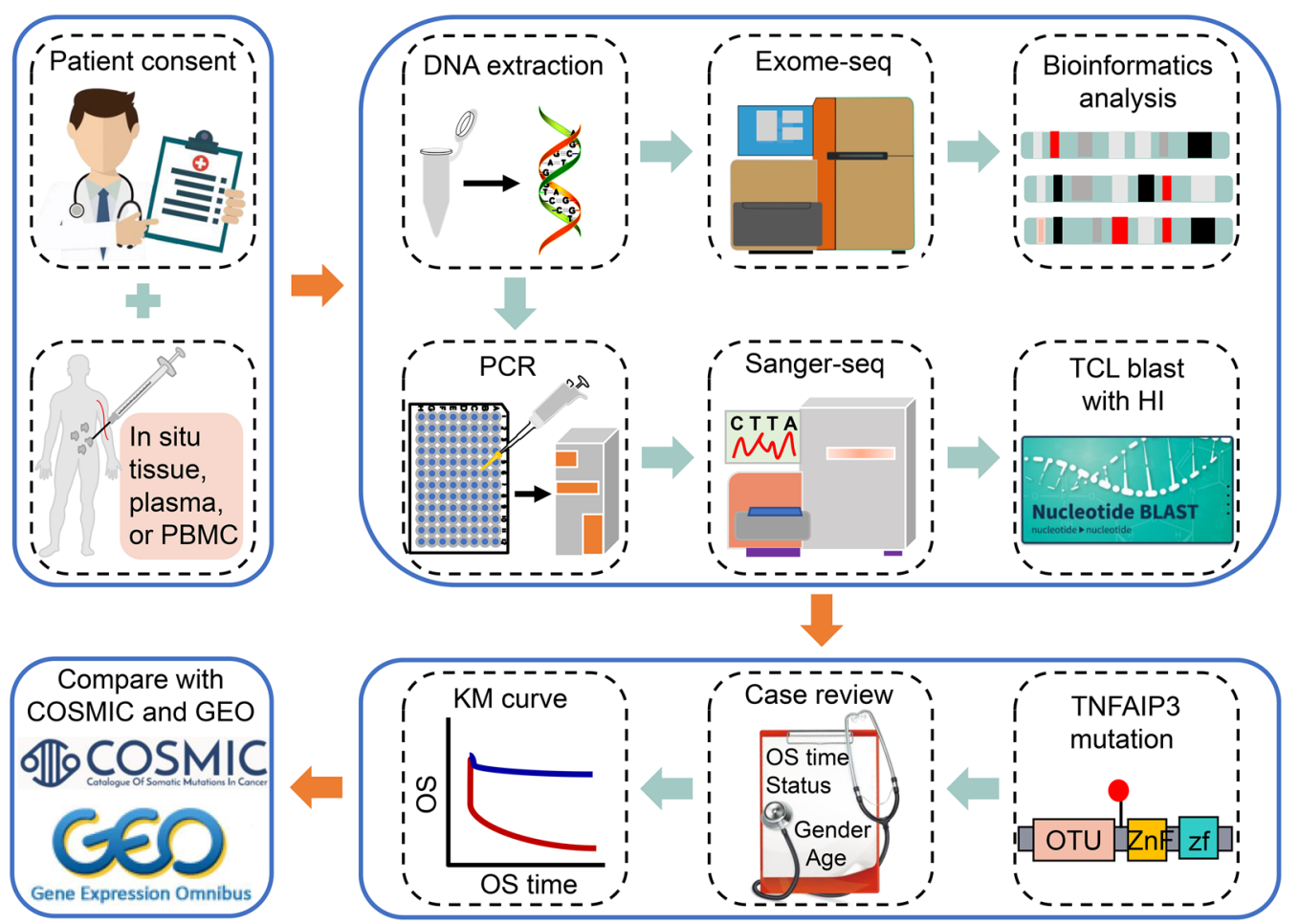

Fig. 1 Schematic diagram of the study. All patients provided informed consent to collecting T-cell lymphoma (TCL) in situ tumor tissue, plasma, or peripheral blood mononuclear cells (PBMCs) for deoxyribonucleic acid (DNA) extraction. Then, the prepared DNA was subjected to exome sequencing for bioinformatics analysis or polymerase chain reaction (PCR) and Sanger sequencing for comparison with healthy individual (HI) sequences, which identified the variant sites and types of TNFAIP3 mutations. Finally, the clinical information of the TCL patients was collected for prognostic analysis, and the TNFAIP3 mutation data from Jinan University (JNU) and Guangdong Provincial People's Hospital (GDPH) were compared to those in the Catalogue of Somatic Mutations in Cancer (COSMIC) and Gene Expression Omnibus (GEO) databases

(Table 1). The TCL subtypes accounting for greater than $10 \%$ of the COSMIC dataset included adult T-cell lymphoma-leukemia (ATLL) (53.7\%), AITL (18.6\%), and CTCL (13.4\%). Notably, the TCL patients in the COSMIC dataset are from the USA (43.6\%), Japan (53.5\%), Australia (1.7\%), Italy (0.9\%), and unknown (0.7\%). The COSMIC and GEO databases are publicly available; thus, approval from the local ethics committee was not required.

\section{Exome sequencing}

Tumor genomic DNA was extracted from tumor biopsies and plasma for library construction, exome sequencing, sequence alignment and processing, and single nucleotide variation (SNV), insertion and deletion (indel), and copy number variation (CNV) detection was performed (Fig. 1). The detailed exome sequencing process was described previously [23].

\section{Polymerase chain reaction (PCR) and Sanger sequencing} The promoter, exons 2-9, and $3^{\prime}$ UTR of the TNFAIP3 gene were amplified by 16 primer pairs, and the primer sequences are shown in Additional file 1: Table S1. PCR was performed as previously described [20, 21]. After the PCR products were Sanger sequenced [20, 21], TCL and $\mathrm{HI}$ sequences were compared using BLAST software (http://blast.ncbi.nlm.nih.gov/Blast.cgi) to the determine variant sites and types of TNFAIP3 mutations.

\section{Statistical analysis}

All statistical analysis was conducted using $\mathrm{R}$ (version 4.0.2, https://www.r-project.org/) [24]. Kaplan-Meier curves were plotted by the $\mathrm{R}$ package "survival", which was compared using the log-rank test $[25,26]$. The $R$ package "survRM2" was also used to determine the restricted mean survival time (RMST) [27]. Differences in two groups of qualitative variables were compared by the Fisher's exact test. A two-tailed $P<0.05$ and a $P<0.1$ were considered statistically significant and a clear trend, respectively. 
Table 1 Clinical characteristics of TCL patients

\begin{tabular}{|c|c|c|c|c|}
\hline Variables & JNU dataset & GDPH dataset & COSMIC dataset & GSE15842 dataset \\
\hline Number & 41 & 79 & 544 & 47 \\
\hline Gender, n (\%) & & & - & - \\
\hline Female & $19(46.3)$ & $30(38.0)$ & - & - \\
\hline Male & $22(53.7)$ & $49(62.0)$ & - & - \\
\hline Age, $y$, mean $\pm S D$ & $48 \pm 19$ & $46 \pm 19$ & - & - \\
\hline \multicolumn{5}{|l|}{ Subtype, n (\%) } \\
\hline AITL & $5(12.2)$ & $18(22.8)$ & $101(18.6)$ & $0(0.0)$ \\
\hline $\mathrm{ALCL}$ & $1(2.4)$ & $6(7.6)$ & $19(3.5)$ & $0(0.0)$ \\
\hline ATLL & $0(0.0)$ & $0(0.0)$ & $292(53.7)$ & $0(0.0)$ \\
\hline CTCL & $1(2.4)$ & $1(1.3)$ & $73(13.4)$ & $0(0.0)$ \\
\hline EATL & $1(2.4)$ & $0(0.0)$ & $0(0.0)$ & $0(0.0)$ \\
\hline HSTCL & $0(0.0)$ & $1(1.3)$ & $3(0.6)$ & $0(0.0)$ \\
\hline MEITL & $0(0.0)$ & $1(1.3)$ & $0(0.0)$ & $0(0.0)$ \\
\hline NKTCL & $15(36.6)$ & $15(19.0)$ & $7(1.3)$ & $0(0.0)$ \\
\hline PTCL-NOS & $5(12.2)$ & $18(22.8)$ & $48(8.8)$ & 47 (100\%) \\
\hline SPTCL & $1(2.4)$ & $1(1.3)$ & $0(0.0)$ & $0(0.0)$ \\
\hline T-LBL & $9(22.0)$ & $18(22.8)$ & $0(0.0)$ & $0(0.0)$ \\
\hline T-LGLL & $3(7.3)$ & $0(0.0)$ & $1(0.2)$ & $0(0.0)$ \\
\hline \multicolumn{5}{|l|}{ Country, n (\%) } \\
\hline China & $41(100)$ & $79(100)$ & $0(0.0)$ & $0(0.0)$ \\
\hline USA & $0(0.0)$ & $0(0.0)$ & $237(43.6)$ & $0(0.0)$ \\
\hline Japan & $0(0.0)$ & $0(0.0)$ & $291(53.5)$ & $0(0.0)$ \\
\hline Australia & $0(0.0)$ & $0(0.0)$ & $9(1.7)$ & $0(0.0)$ \\
\hline Italy & $0(0.0)$ & $0(0.0)$ & $5(0.9)$ & $0(0.0)$ \\
\hline Germany & $0(0.0)$ & $0(0.0)$ & $0(0.0)$ & $47(100 \%)$ \\
\hline Unknown & $0(0.0)$ & $0(0.0)$ & $4(0.7)$ & $0(0.0)$ \\
\hline Detection method & $P C R+$ sanger sequencing & Exome sequencing & - & SNP array \\
\hline Covered area & Non-CDS and CDS & CDS & CDS & - \\
\hline Follow up, m, median (range) & $23.37(0.23$ to 157.83$)$ & - & - & - \\
\hline Status (alive/dead), n & $22 / 19$ & - & - & - \\
\hline
\end{tabular}

The bold values indicated that the proportion of TCL subtypes accounting for more than $10 \%$ of the sample

AITL angioimmunoblastic T-cell lymphoma, $A L C L$ anaplastic large cell lymphoma, $A T L L$ adult T-cell lymphoma-leukemia, $C D S$ coding sequence, COSMIC catalogue of somatic mutations in cancer, CTCL cutaneous T-cell lymphoma, EATL enteropathy-associated T-cell lymphoma, GDPH Guangdong Provincial People's Hospital, HSTCL hepatosplenic T-cell lymphoma, JNU Jinan University, MEITL monomorphic epitheliotropic intestinal T-cell lymphoma, NGS next-generation sequencing, NKTCL NK-T-cell lymphoma, PCR polymerase chain reaction, PTCL-NOS peripheral T-cell lymphoma, not otherwise specified, SNP single nucleotide polymorphism, SPTCL subcutaneous panniculitis-like T-cell lymphoma, T-LBL T-lymphoblastic lymphoma, T-LGLL T-cell large granular lymphocytic leukemia

${ }^{a}$ Due to rounding, not all percentages total $100 \%$

\section{Results}

\section{Mutation pattern of TNFAIP3 in TCL}

In COSMIC samples, all of the TNFAIP3 mutations are found in the CDS region with an overall mutation frequency of $2.2 \%$ (12/544) in TCL (Table 2; Fig. 2A). The CTCL mutation frequency was relatively high, reaching $6.3 \%$, and no TNFAIP3 mutations were found in anaplastic large cell lymphoma (ALCL), NKTCL, hepatosplenic T-cell lymphoma (HSTCL), or large granular $\mathrm{T}$ lymphocyte leukemia (T-LGLL) (Fig. 2A). We further analyzed the types of mutations and found that missense, frameshift insertions, nonsense, and frameshift deletions mainly occurred in the TNFAIP3 gene, and the single base substitutions $\mathrm{C}>\mathrm{T}$ (positions g.811 and g.1166) and $\mathrm{A}>\mathrm{C}$ (position g.1939) were significantly prominent (Fig. 2B, C). However, in samples obtained from our center (GDPH and JNU datasets), there were no mutations detected in the TNFAIP3 CDS region by highthroughput sequencing, PCR, or Sanger sequencing. However, we did detect mutations in non-CDS regions in TCL samples in the JNU dataset by both PCR and Sanger sequencing. Interestingly, we found that $14.6 \%$ (6/41) of TCL patients had a TNFAIP3 mutation in the non-CDS region (Table 2; Fig. 3A, left panel). Among 
Table 2 Clinical characteristics of T-cell lymphoma patients with a TNFAIP3 mutation

\begin{tabular}{|c|c|c|c|c|c|c|c|c|}
\hline Sample ID & Histological subtype & Age, $y$ & Gender & Country & Mutation sites & $\begin{array}{l}\text { TNFAIP3 } \\
\text { mutation }\end{array}$ & $\begin{array}{l}\text { OS time } \\
\text { (months) }\end{array}$ & Clinical outcome \\
\hline \multicolumn{9}{|c|}{ COSMIC dataset } \\
\hline Patient 1 & PTCL-NOS & $<60$ & Female & USA & CDS & g.305 A>G & - & Not relapse \\
\hline Patient 2 & PTCL-NOS & $\geq 60$ & Female & USA & CDS & g.1939 A>C & - & Not relapse \\
\hline Patient 3 & PTCL-NOS & - & - & USA & CDS & g.1939 A>C & - & Not relapse \\
\hline Patient 4 & CTCL & $\geq 60$ & Male & USA & CDS & $\begin{array}{l}\text { g.1307G }>A \text {, g.796dup, and } \\
\text { g. } 732 \text { C >A }\end{array}$ & - & Alive \\
\hline Patient 5 & AITL & - & Female & USA & CDS & g.811 C>T & - & Alive \\
\hline Patient 6 & $\mathrm{CTCL}$ & 67 & Male & USA & CDS & g.-15-1829_-15-1814del & - & - \\
\hline Patient 7 & ATLL & 56 & Male & Japan & CDS & g.318dup & - & - \\
\hline Patient 8 & CTCL & - & Female & USA & CDS & g.380T $>\mathrm{G}$ & - & - \\
\hline Patient 9 & ATLL & - & - & Japan & CDS & g.1616_1617insT & - & - \\
\hline Patient 10 & ATLL & - & - & Japan & CDS & g.1439_1440dup & - & - \\
\hline Patient 11 & ATLL & 79 & Female & Japan & CDS & g.1667dup & - & - \\
\hline Patient 12 & ATLL & - & - & Japan & CDS & g.1166C>T & - & - \\
\hline \multicolumn{9}{|c|}{ GSE15842 dataset } \\
\hline Patient 13 & PTCL-NOS & - & - & Germany & Intron & rs582757 & - & - \\
\hline Patient 14 & PTCL-NOS & - & - & Germany & Intron & rs582757 & - & - \\
\hline \multicolumn{9}{|l|}{ JNU dataset } \\
\hline Patient 15 & T-LBL & 69 & Female & China & Promoter & g.3918 C>T & 98.9 & Death \\
\hline Patient 16 & CTCL & 36 & Male & China & Promoter & g.3918 C>T and g. 3637 T >A & 58.8 & Death \\
\hline Patient 17 & T-LBL & 19 & Male & China & $3^{\prime} U T R$ & g.3869 C>G & 94.3 & Alive \\
\hline Patient 18 & T-LGLL & 27 & Male & China & Intron & g.13751 A>C & 97.1 & Alive \\
\hline Patient 19 & T-LGLL & 40 & Female & China & Intron & g.13751 A >C & 118.5 & Alive \\
\hline Patient 20 & T-LGLL & 50 & Female & China & Intron & $\begin{array}{l}\text { g.13751 A>C, g.14244 C>T, and } \\
\text { g.11822T>C }\end{array}$ & 102.4 & Alive \\
\hline
\end{tabular}

AITL angioimmunoblastic T-cell lymphoma, ATLL adult T-cell lymphoma-leukemia, CDS coding sequence, CTCL cutaneous T-cell lymphoma, JNU Jinan University, OS overall survival, PTCL-NOS peripheral T-cell lymphoma, not otherwise specified, T-LBL T-lymphoblastic lymphoma, $T$-LGLL T-cell large granular lymphocytic leukemia, UTR untranslated region

these mutations, $\mathrm{C}>\mathrm{T}$ alterations (positions g.3918 and g.14244) and $\mathrm{A}>\mathrm{C}$ (position g.13751) were the most common in TCL (Fig. 3A, right panel and 3B). Further subgroup analysis revealed that the TCL subtypes with a TNFAIP3 mutation included 3/6 T-LGLL, 2/6 T-LBL, and 1/6 CTCL (Table 2). Notably, 4.3\% (2/47) of PTCLNOS patients had a TNFAIP3 mutation in the non-CDS region in the GSE15842 dataset (Table 2).

\section{TNFAIP3 mutation correlates with favorable OS for TCL patients}

To further investigate the relationship between TNFAIP3 mutation and OS for TCL patients, we conducted a prognostic analysis. Interestingly, there was a clear trend where TCL patients harboring a TNFAIP3 mutation in non-CDS regions in our center (JNU dataset) were associated with favorable OS, although the data were not yet statistically significant at this point [hazard ratio $(\mathrm{HR})=0.29$; 95\% confidence interval $(\mathrm{CI}): 0.07$ to 1.31 ; 5 -year OS rate: $83 \%$ vs. $44 \%$; $P=0.089$ ] (Fig. $4 \mathrm{~A}$ ). Moreover, RMST was used to evaluate the performance of the
Kaplan-Meier curve. As shown in Fig. 4B, the 5-year RMST for TCL patients with or without a TNFAIP3 mutation in the JNU dataset was 59.80 (95\% CI 59.44 to 60.16 ) and 36.98 (95\% CI 28.18 to 45.78 ) months, respectively. To clarify the relationship between TNFAIP3 mutation and TCL subtypes, we next conducted a correlation analysis. As shown in Fig. $4 \mathrm{C}$, there was a clear trend indicating that T-LGLL patients were associated with favorable OS compared to non-T-LGLL patients (5-year OS rate: $100 \%$ vs. $45 \%$; $P=0.061$ ), although the data were not yet statistically significant at this point. Interestingly, TNFAIP3 mutation was positively correlated with T-LGLL $(P=0.002$, Fig. 4D).

\section{Discussion}

TNFAIP3 is a well-known negative regulator of the NF- $\kappa B$ signaling pathway, and mutations in this gene are usually observed in cancer, including hematological malignancies, which affect the clinical outcome of patients [13, 28-30]. Additionally, the mutation of TNFAIP3 has been widely studied in B-cell lymphoma 


\section{COSMIC database}

A

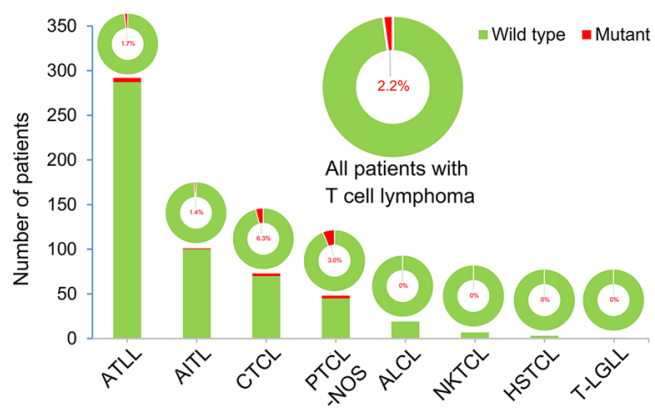

C
B

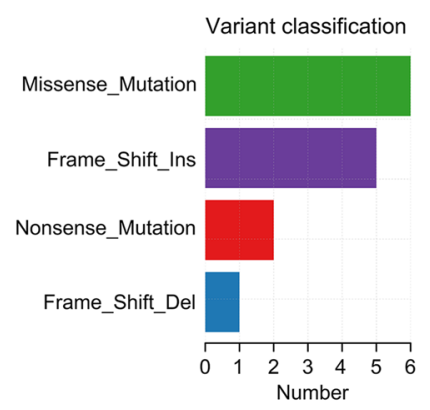

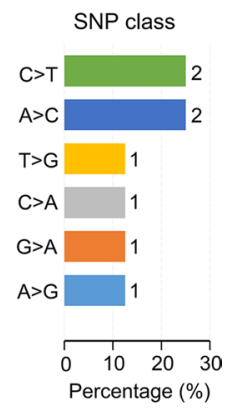

COSMIC database

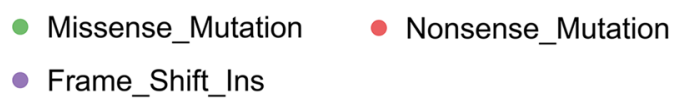

- Missense_Mutation

- Frame_Shift_Ins

- Nonsense_Mutation

C Frame_Shift_I "i
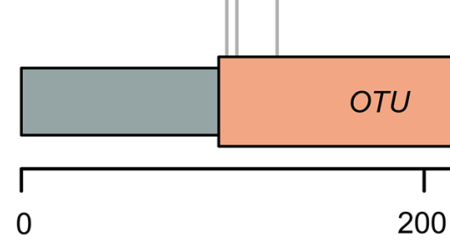

200
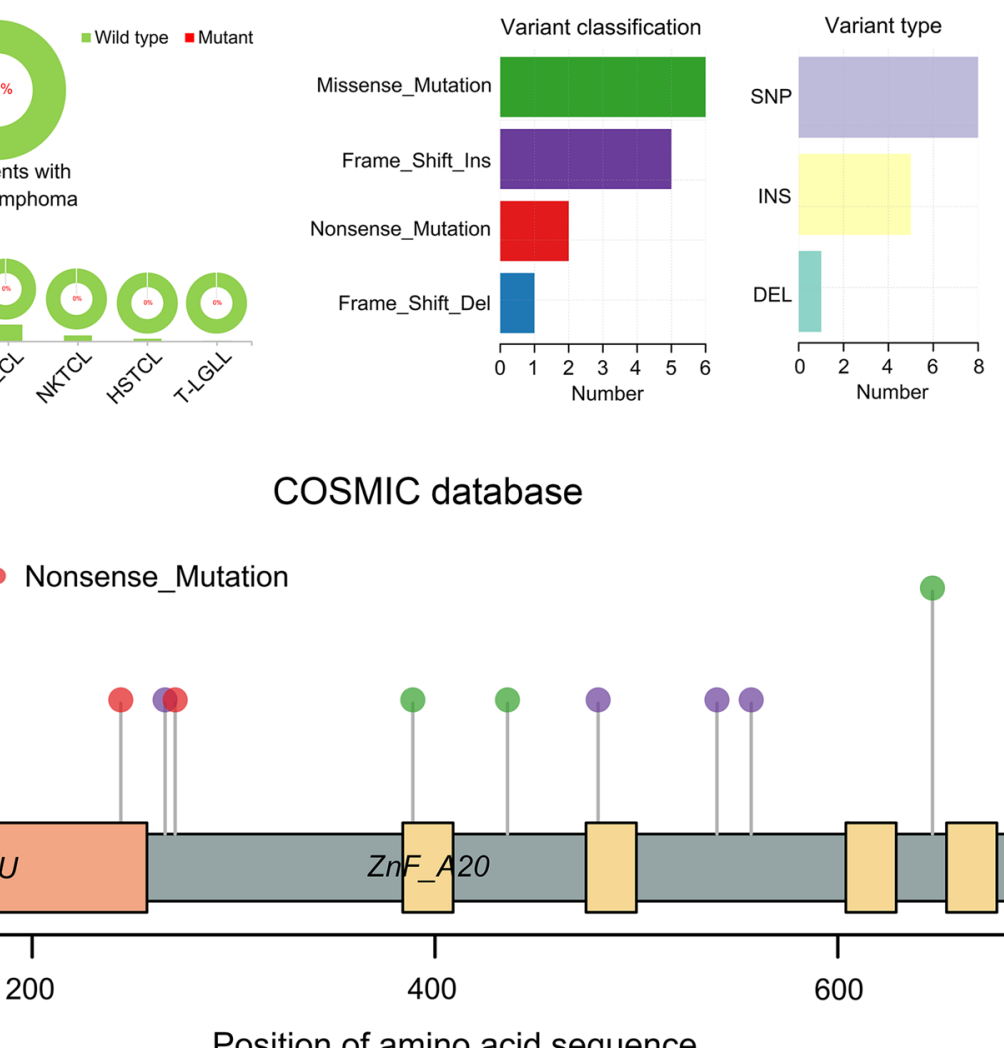

\section{Position of amino acid sequence}

Fig. 2 The variant sites, types of TNFAIP3 mutations, and percentage of mutations in the COSMIC dataset. A B B The percentage of TNFAIP3 mutations in TCL patients (A), and variant classification, variant type, and single nucleotide polymorphism (SNP) class (B) in the COSMIC database. C The package "maftools" in R (version 4.0.2, https://www.r-project.org/) was used to obtain the variant sites in TNFAIP3 in the COSMIC database

$[13,16,31]$, but its mutation pattern and impact on the prognosis of TCL patients are little known [15]. Because there are few studies regarding the alteration of TNFAIP3 in $\mathrm{T}$ cell malignancies in China. In this study, we firstly focused on comparing the differences in CDS mutations of TNFAIP3 between China and other countries. Interestingly, we observed this different pattern of TNFAIP3 mutation in CDS in Chinese TCL patients. We suggested that it may be due to the differences in population distribution characteristics. This suggestion is also based on our previous finding on the different SNP distribution of TNFAIP3 in CDS in patients with rheumatoid arthritis in comparison with patients from other countries [32]. Further confirmation for this alteration will be done in another cohort of $\mathrm{T}$ cell lymphoma samples from China. Notably, $2.2 \%$ of TCL patients had mutations in the CDS region of TNFAIP3 in the COSMIC dataset, but none were found in our clinical center's datasets. Interestingly, we further examined the non-CDS region of TNFAIP3 and found that $14.6 \%$ of TCL patients had mutations in the JNU dataset. We did try to find the feature of TNFAIP3 mutation in the non-CDS region from different datasets. The only one dataset with SNP data of TNFAIP3 is the GSE15842 dataset. 4.3\% of TCL patients had a TNFAIP3 mutation in the non-CDS region. However, there is no prognostic information in the GSE15842 dataset for us to analyze the correlation between the nonCDS mutation of TNFAIP3 and the prognosis of TCL patients. There was a report describing bi- and monoallelic deletions of TNFAIP3 in a high proportion of CTCL patients [18]. Importantly, our work uncovered that $6.3 \%$ of CTCL patients have TNFAIP3 mutations, and one of which was a deletion mutation found in the COSMIC database. Moreover, although a few studies have shown that TNFAIP3 deletion is detected in $18-35 \%$ of extranodal NKTCL (ENKTCL) patients $[15,19]$, no mutations in TNFAIP3 in this subtype were found in this study. Furthermore, single base-pair mutations were detected 

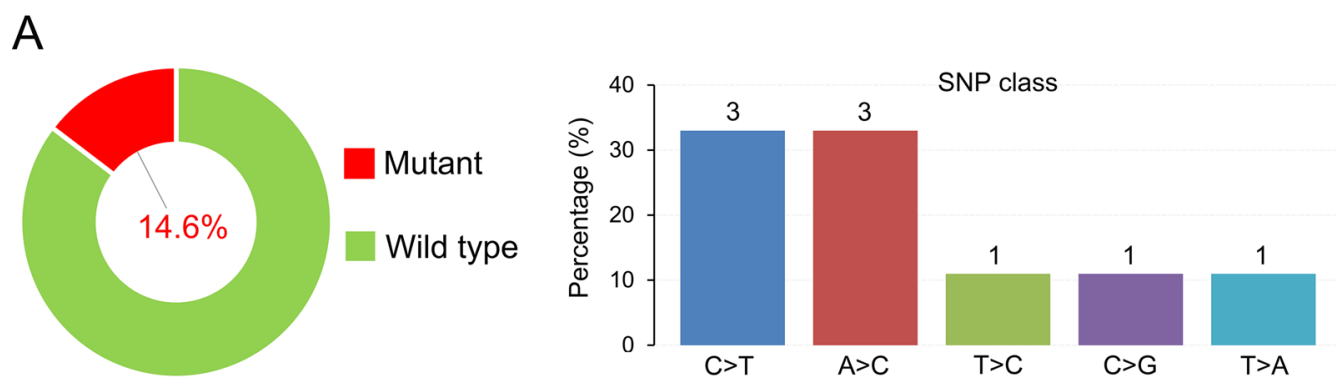

B

rs661561

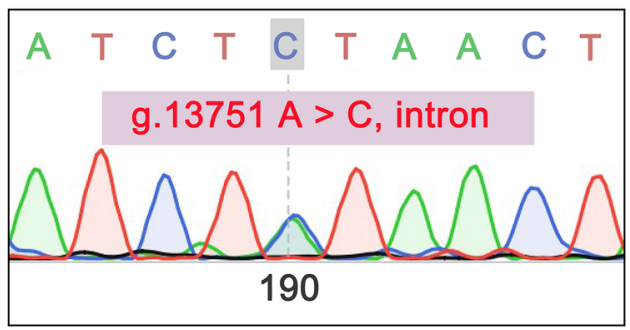

rs598493

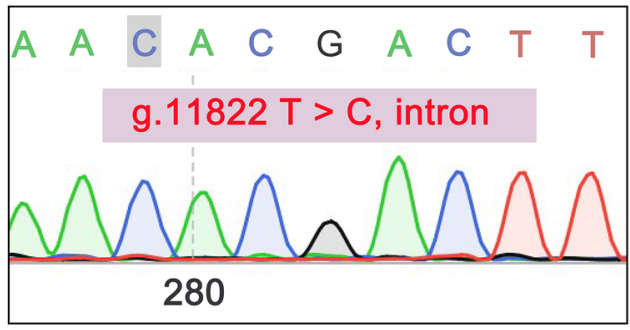

rs5029924

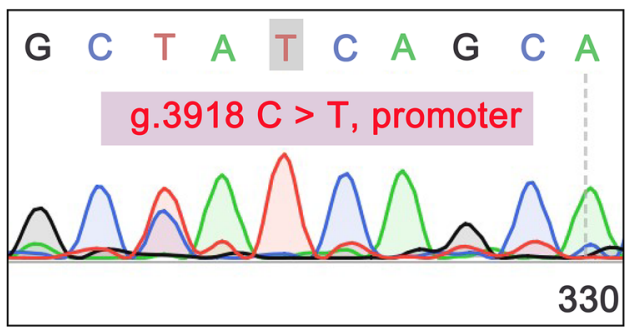

\section{rs582757}

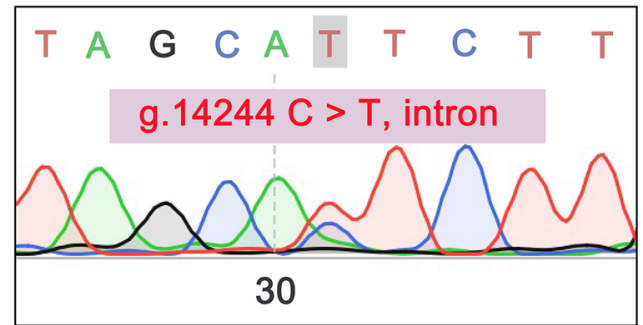

g.3637 T>A

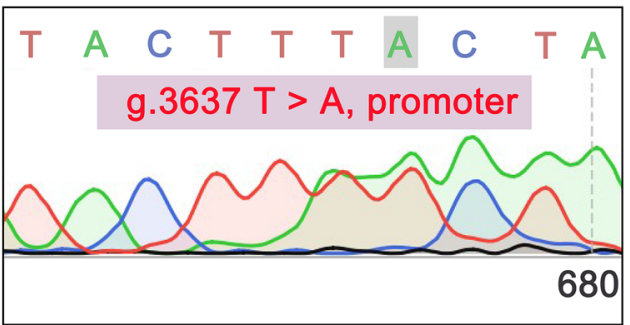

g.3869 C>G

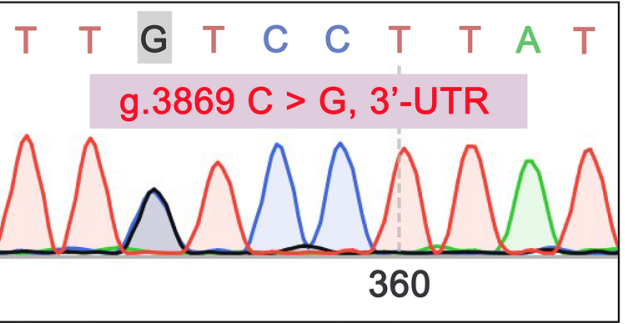

Fig. 3 The TNFAIP3 mutation pattern in the JNU dataset. A Percentage of TNFAIP3 mutations (left panel) and SNP class (right panel) in TCL patients from the JNU dataset. B Representative variant sites in the TNFAIP3 introns, promoter, and 3' untranslated region (3'UTR) were obtained from the SNP database (https://www.ncbi.nlm.nih.gov/snp/)

rather than deletions in the $3^{\prime} \mathrm{UTR}$, promoter, and intron regions using PCR, Sanger, and exome sequencing in our clinical center's datasets. Finally, TNFAIP3 mutation significantly correlated with T-LGLL. This study identified the distribution characteristics of TNFAIP3 mutations in TCL subtypes. Recently, researchers have explored the prognostic value of TNFAIP3 deletion in patients with
ENKTCL, but inconsistent results have been found. Ahn et al. reported that TNFAIP3 deletions were associated with poor progression-free survival (PFS) in ENKTCL patients, while Liu et al. suggested that heterozygous deletions in TNFAIP3 predicted favorable OS in ENKTCL $[15,19]$. In previous reports, TNFAIP3 negatively regulates the activity of NF- $\mathrm{kB}$ by up-regulating the 
A

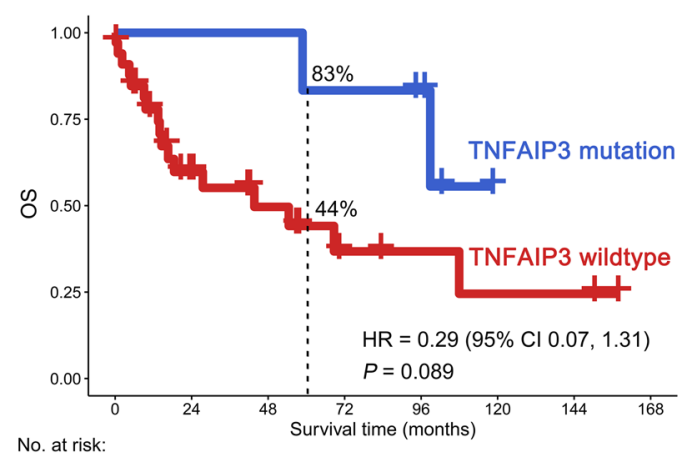

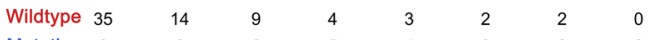

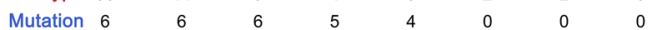

C

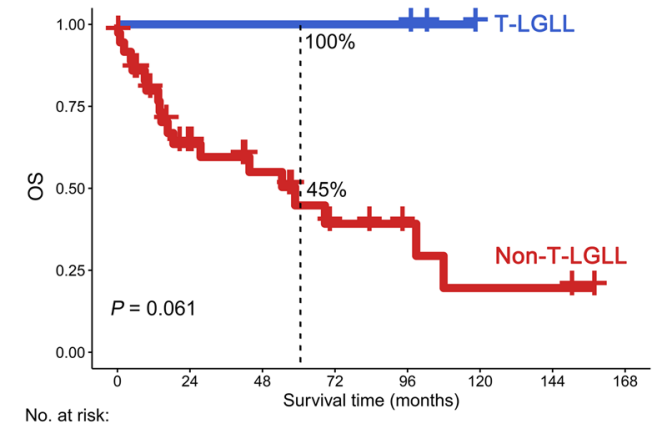

$\begin{array}{lllllllll}\text { Non-T-LGLL } 38 & 17 & 12 & 6 & 4 & 2 & 2 & 0\end{array}$

$\begin{array}{cccccccc}\text { T-LGLL } 3 & 3 & 3 & 3 & 3 & 0 & 0 & 0\end{array}$

Fig. 4 Overall survival (OS) analysis of TNFAIP3 mutaions in TCL patients in the JNU dataset. A, B Kaplan-Meier curve (A) and 5-year restricted mean survival time (RMST) (B) based on the status of TNFAIP3 mutations are shown. C Overall survival (OS) analysis of T-cell large granular lymphocytic leukemia (T-LGLL) and non-T-LGLL patients. D The number (left panel) and percentage (right panel) of TNFAIP3 mutations in T-LGLL and non-T-LGLL patients
B
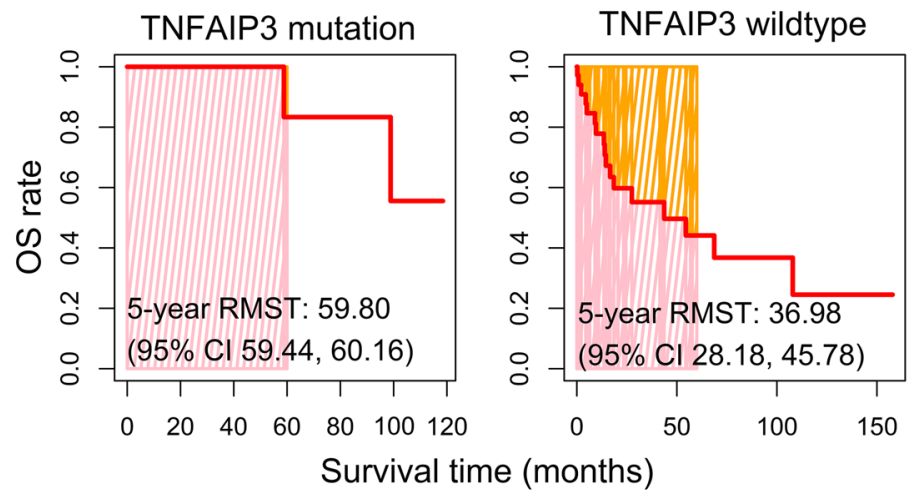

D
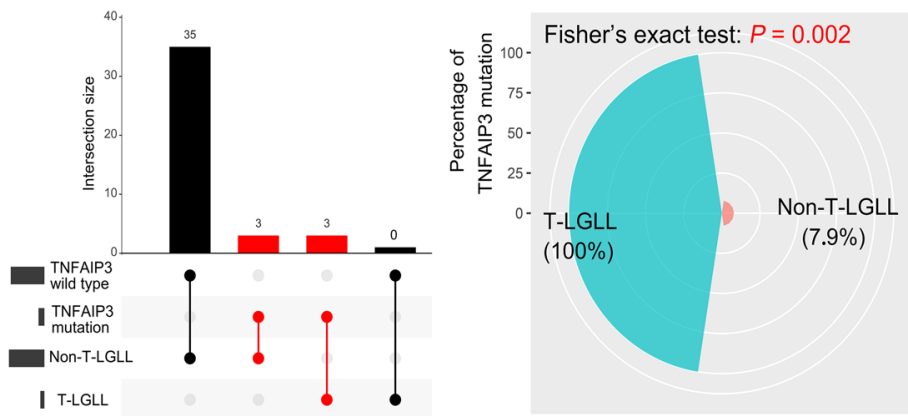

$\frac{1}{3020100}$ expression level to inhibit tumor development. While, TNFAIP3 was also thought to be an oncogene in numbers of solid tumors such as ductal carcinoma in situ, gastric carcinoma, basal-like breast cancer [33-35]. Moreover, TNFAIP3 promotes CD4 $+\mathrm{T}$ cell survival by restricting ubiquitination of the mTOR (mechanistic target of rapamycin kinase) complex and increased autophagy [36]. In this study, we found that TCL patients with TNFAIP3 mutations were associated with favorable OS. However, we had not done the experiment to characterize the role of the mutated TNFAIP3 in TCL cells. Therefore, we can only report the first finding which may be a novel biomarker for predicting the clinical outcome of TCL. And we will further explore the role of such mutations in malignant $\mathrm{T}$ cells to characterize their function and the related mechanism of the patients carrying non-CDS mutation of TNFAIP3 with favorable OS. It is known that T-LGLL patients have a favorable prognosis with a 30 -year OS rate of up to $70 \%[37,38]$, which is consistent with this study. Interestingly, the results of this study indicated that mutant-TNFAIP3 significantly correlates with T-LGLL. Indeed, it would be perfect, that we could show the validation results either from the database or from another cohort of clinical samples. We did try to obtain another dataset from the publicly available database to validate our results. Unfortunately, there is no dataset containing both prognosis and TNFAIP3 mutation information for us to further analyze and validate. Thus, on the other hand, our data may be provided the first information of TNFAIP3 mutation in non-CDS linking to prognosis of TCL, which may be a potential biomarker for predict the prognosis of patients with TCL. Therefore, a larger cohort samples are needed to further validate the relationship between TNFAIP3 mutation and favorable OS in the future.

The difference between the TNFAIP3 mutation pattern in the COSMIC dataset and our clinical center's datasets may be due to the following reasons. First, the population 
distribution characteristics of TCL subtypes will lead to differences in the TNFAIP3 mutation detection rate. In the GDPH and JNU datasets, NKTCL, T-LBL, AITL, and PTCL-NOS comprised the TCL subsets with the highest percentages, while in the COSMIC dataset, they were ATLL, AITL, and CTCL. Second, the TCL patients in the three datasets come from different countries, which may be a reason why the TNFAIP3 mutation patterns were inconsistent. The TCL patients in the JNU and GDPH datasets all come from China, while in the COSMIC dataset, they come from the USA, Japan, Australia, and Italy. Therefore, it is necessary to further confirm the role of mutant TNFAIP3 in the evaluation of OS for TCL patients according to the distribution characteristics of TCL subtypes in different countries.

\section{Conclusions}

We for the first time demonstrate a low mutation frequency of TNFAIP3 in TCL, and the mutations were primarily located in the non-CDS region in Chinese patients. Notably, mutant TNFAIP3 might be a predictor of favorable OS in TCL patients, which might complement current risk stratification of Chinese TCL patients.

\begin{abstract}
Abbreviations
AITL: Angioimmunoblastic T-cell lymphoma; ALCL: Anaplastic large cell lymphoma; ATLL: Adult T-cell lymphoma-leukemia; CDS: Coding sequence; Cl: Confidence interval; COSMIC: Catalogue of somatic mutations in cancer; CTCL: Cutaneous T-cell lymphoma; EATL: Enteropathy-associated T-cell lymphoma; GDPH: Guangdong Provincial People's Hospital; HI: Healthy individual; HSTCL: Hepatosplenic T-cell lymphoma; HR: Hazard ratio; Indel: Insertion and deletion; IPI: International prognosis index; JUN: Jinan University; NGS: Next-generation sequencing; NKTCL: NK-T-cell lymphoma; OS: Overall survival; PCR: Polymerase chain reaction; PBMC: Peripheral blood mononuclear cell; PTCL-NOS: Peripheral T-cell lymphoma, not otherwise specified; RMST: Restricted mean survival time; SNV: Single nucleotide variation; SPTCL: Subcutaneous panniculitis-like T-cell lymphoma; T-LGLL:T-cell large granular lymphocytic leukemia; TCL:T-cell lymphoma; TNFAIP3: Tumor necrosis factor alpha-induced protein 3.
\end{abstract}

\section{Supplementary Information}

The online version contains supplementary material available at https://doi. org/10.1186/s12935-021-02191-5.

Additional file 1: Table S1. PCR primers for TNFAIP3.

\section{Acknowledgements}

Not applicable.

\section{Authors' contributions}

CTC interpreted the data and wrote the manuscript. ZC and LH collected the clinical information and helped to write the manuscript. LLZ and LHZ performed the experiments. SCL collected and interpreted the clinical data. GXL, LH and WYL diagnosed and treated the patients and provided clinical information. CWZ edited and reviewed the manuscript. YQL contributed to the concept development, study design, and edited the manuscript. All authors read and approved the final manuscript.
}

\section{Funding}

This work was supported by grants from the Intergovernmental International Cooperation on Scientific and Technological Innovation project of Chinese Ministry of Science and Technology (No. 2017YFE0131600), the Guangdong Science and Technology Project (Nos. 2020A0505100042 and 2016A020215123), and the major program of Summit Project, Guangdong Province High-level Hospital Construction Project of Guangdong Provincial People's Hospital, Guangdong Academy of Medical Sciences (DFJH2020025).

\section{Availability of data and materials}

The COSMIC data used in this study were acquired from the COSMIC database (https://cancer.sanger.ac.uk/cosmic). The datasets used and/or analyzed during the current study are available from the corresponding author on reasonable request.

\section{Declarations}

Ethics approval and consent to participate

This study was performed according to the Declaration of Helsinki principles and approved by the Ethics Committee of the First Affiliated Hospital of Jinan University. All participants provided written informed consent.

\section{Consent for publication}

Not applicable.

\section{Competing interests}

The authors declare that they have no competing interests.

\section{Author details}

${ }^{1}$ Institute of Hematology, School of Medicine, Key Laboratory for Regenerative Medicine of Ministry of Education, Jinan University, Guangzhou 510632, China. ${ }^{2}$ Department of Lymphoma, Guangdong Provincial People's Hospital, Guangdong Academy of Medical Sciences, School of Medicine, South China University of Technology, Guangzhou, China. ${ }^{3}$ Department of Hematology, Nanfang Hospital, Southern Medical University, Guangzhou, China. ${ }^{4}$ Department of Rheumatism and Immunology, First Affiliated Hospital, Jinan University, Guangzhou, China. ${ }^{5}$ Department of Hematology, First Affiliated Hospital, Jinan University, Guangzhou, China.

Received: 9 June 2021 Accepted: 3 September 2021

Published online: 15 September 2021

\section{References}

1. Zain JM. Aggressive T-cell lymphomas: 2019 updates on diagnosis, risk stratification, and management. Am J Hematol. 2019;94(8):929-46.

2. de Mel S, Hue SS, Jeyasekharan AD, Chng WJ, Ng SB. Molecular pathogenic pathways in extranodal NK/T cell lymphoma. J Hematol Oncol. 2019:12(1):33.

3. Zu Y. Aptamer technology: a new approach to treat lymphoma? Blood Sci. 2020;2(1):11-5.

4. Zhang S, Li M, Yuan F, Chen L, Mi R, Wei X, Song Y, Yin Q. The predictive value of dynamic monitoring of peripheral blood lymphocyte to monocyte ratio in patients with extranodal NK/T cell lymphoma. Cancer Cell Int. 2019;19:272.

5. Liu Y, Wang X, Deng L, Ping L, Shi Y, Zheng W, Lin N, Wang X, Tu M, Xie $Y$, et al. ITK inhibition induced in vitro and in vivo anti-tumor activity through downregulating TCR signaling pathway in malignant T cell lymphoma. Cancer Cell Int. 2019;19:32.

6. Jiang M, Bennani NN, Feldman AL. Lymphoma classification update: T-cell lymphomas, Hodgkin lymphomas, and histiocytic/dendritic cell neoplasms. Expert Rev Hematol. 2017;10(3):239-49.

7. Toner K, Bollard CM, Dave H. T-cell therapies for T-cell lymphoma. Cytotherapy. 2019;21(9):935-42.

8. Chihara D, Fanale MA, Miranda RN, Noorani M, Westin JR, Nastoupil LJ, Hagemeister FB, Fayad LE, Romaguera JE, Samaniego F, et al. The survival outcome of patients with relapsed/refractory peripheral T-cell lymphoma-not otherwise specified and angioimmunoblastic T-cell lymphoma. Br J Haematol. 2017;176(5):750-8. 
9. Chen SY, Yang Y, Qi SN, Wang Y, Hu C, He X, Zhang LL, Wu G, Qu BL, Qian $L T$, et al. Validation of nomogram-revised risk index and comparison with other models for extranodal nasal-type NK/T-cell lymphoma in the modern chemotherapy era: indication for prognostication and clinical decision-making. Leukemia. 2021;35(1):130-42.

10. Ellin F, Maurer MJ, Srour L, Farooq U, Jerkeman M, Connors JM, Smedby KE, Bennani NN, Ansell SM, Slack GW, et al. Comparison of the NCCN-IPI, the IPI and PIT scores as prognostic tools in peripheral T-cell lymphomas. Br J Haematol. 2019:186(3):e24-7.

11. Kihara R, Watanabe T, Yano T, Uike N, Okamura S, Kawano F, Hanada S, Sunami K, Inoue N, Sawamura M, et al. Prognosis of mature T cell lymphoma is poorer than that of diffuse large B cell lymphoma in IPI lowrisk group, but not in intermediate- and high-risk groups. Int J Hematol. 2013;97(1):98-102.

12. Pujari R, Hunte R, Khan WN, Shembade N. A20-mediated negative regulation of canonical NF-KB signaling pathway. Immunol Res. 2013;57(1-3):166-71.

13. Zhang F, Yang L, LiY. The role of A20 in the pathogenesis of lymphocytic malignancy. Cancer Cell Int. 2012;12(1):44.

14. Yu Z, Chen C, Xiao Y, Chen X, Guo L, Tan G, Huang G, Luo W, Zhou M, Li Y, et al. Abnormal miR-214/A20 expression might play a role in T cell activation in patients with aplastic anemia. Blood Sci. 2020;2(3):100-5.

15. Ahn H, Yang JM, Jeon YK, Paik JH. Clinicopathologic implications of TNFAIP3/A20 deletions in extranodal NK/T-cell lymphoma. Genes Chromosom Cancer. 2018;57(5):231-9.

16. Paik JH, Go H, Nam SJ, Kim TM, Heo DS, Kim CW, Jeon YK. Clinicopathologic implication of A20/TNFAIP3 deletion in diffuse large B-cell lymphoma: an analysis according to immunohistochemical subgroups and rituximab treatment. Leuk Lymphoma. 2013;54(9):1934-41.

17. Novak U, Rinaldi A, Kwee I, Nandula SV, Rancoita PM, Compagno M, Cerri M, Rossi D, Murty W, Zucca E, et al. The NF-kB negative regulator TNFAIP3 (A20) is inactivated by somatic mutations and genomic deletions in marginal zone lymphomas. Blood. 2009;113(20):4918-21.

18. Braun FC, Grabarczyk P, Möbs M, Braun FK, Eberle J, Beyer M, Sterry W, Busse F, Schröder J, Delin M, et al. Tumor suppressor TNFAIP3 (A20) is frequently deleted in Sézary syndrome. Leukemia. 2011;25(9):1494-501.

19. Liu F, Zheng JP, Wang L, Zhao DH, Li MY, Wang YM, Liu Y, Ma J, Zeng NY, Liu HX, et al. Activation of the NF-KB pathway and heterozygous deletion of TNFAIP3 (A20) confer superior survival in extranodal natural killer/T-cell lymphoma, nasal type. Am J Clin Pathol. 2019;152(2):243-52.

20. Zhu L, Zhang F, Shen Q, Chen S, Wang X, Wang L, Yang L, Wu X, Huang S, Schmidt CA, et al. Characteristics of A20 gene polymorphisms in T-cell acute lymphocytic leukemia. Hematology. 2014;19(8):448-54.

21. Zhou L, Zheng H, Huang X, Zhu L, Wu S, Zeng C, Yang L, Chen S, Luo G, Du X, et al. Different genetic alteration of A20 in a Sézary syndrome case with Va2-Ja22 T cell clone. Asia Pac J Clin Oncol. 2018;14(2):e116-23.

22. Vardiman JW. The World Health Organization (WHO) classification of tumors of the hematopoietic and lymphoid tissues: an overview with emphasis on the myeloid neoplasms. Chem Biol Interact. 2010;184(1-2):16-20.

23. Chen C, Liu S, Jiang X, Huang L, Chen F, Wei X, Guo H, Shao Y, Li Y, Li W. Tumor mutation burden estimated by a 69-gene-panel is associated with overall survival in patients with diffuse large B-cell lymphoma. Exp Hematol Oncol. 2021;10(1):20.

24. Li K, Chen C, Gao R, Yu X, Huang Y, Chen Z, Liu Z, Chen S, Luo G, Huang X, et al. Inhibition of BCL11B induces downregulation of PTK7 and results in growth retardation and apoptosis in T-cell acute lymphoblastic leukemia. Biomark Res. 2021;9(1):17.
25. Chen C, Xu L, Gao R, Wang S, Zhang Y, Wang C, Zeng C, Li Y. Transcriptome-based co-expression of BRD4 and PD-1/PD-L1 predicts poor overall survival in patients with acute myeloid leukemia. Front Pharmacol. 2020;11:582955.

26. Chen C, Liang C, Wang S, Chio CL, Zhang Y, Zeng C, Chen S, Wang C, Li Y. Expression patterns of immune checkpoints in acute myeloid leukemia. J Hematol Oncol. 2020;13(1):28.

27. Chen C, Chio CL, Zeng H, Li Y. High expression of CD56 may be associated with favorable overall survival in intermediate-risk acute myeloid leukemia. Hematology. 2021;26(1):210-4.

28. Audil HY, Hampel PJ, Van Dyke DL, Achenbach SJ, Rabe KG, Smoley SA, Call TG, Ding W, Shi M, Hanson CA, et al. The prognostic significance of del6q23 in chronic lymphocytic leukemia. Am J Hematol. 2021;96(6):E203-6.

29. Wenzl K, Manske MK, Sarangi V, Asmann YW, Greipp PT, Schoon HR, Braggio E, Maurer MJ, Feldman AL, Witzig TE, et al. Loss of TNFAIP3 enhances MYD88(L265P)-driven signaling in non-Hodgkin lymphoma. Blood Cancer J. 2018:8(10):97.

30. Ungerbäck J, Belenki D, Jawad ul-Hassan A, Fredrikson M, Fransén K, Elander N, Verma D, Söderkvist P. Genetic variation and alterations of genes involved in NFKB/TNFAIP3- and NLRP3-inflammasome signaling affect susceptibility and outcome of colorectal cancer. Carcinogenesis. 2012;33(11):2126-34.

31. Vela V, Juskevicius D, Gerlach MM, Meyer P, Graber A, Cathomas G, Dirnhofer S, Tzankov A. High throughput sequencing reveals high specificity of TNFAIP3 mutations in ocular adnexal marginal zone B-cell lymphomas. Hematol Oncol. 2020;38(3):284-92.

32. Zhu L, Wang L, Wang X, Zhou L, Liao Z, Xu L, Wu H, Ren J, Li Z, Yang L, et al. Characteristics of A20 gene polymorphisms and clinical significance in patients with rheumatoid arthritis. J Transl Med. 2015;13:215.

33. Yang M, Yu X, Li X, Luo B, Yang W, Lin Y, Li D, Gan Z, Xu J, He T. TNFAIP3 is required for FGFR1 activation-promoted proliferation and tumorigenesis of premalignant DCIS.COM human mammary epithelial cells. Breast Cancer Res. 2018;20(1):97.

34. Du B, Liu M, Li C, Geng X, Zhang X, Ning D, Liu M. The potential role of TNFAIP3 in malignant transformation of gastric carcinoma. Pathol Res Pract. 2019;215(8):152471

35. Lee JH, Jung SM, Yang KM, Bae E, Ahn SG, Park JS, Seo D, Kim M, Ha J, Lee J, et al. A20 promotes metastasis of aggressive basal-like breast cancers through multi-monoubiquitylation of Snail1. Nat Cell Biol. 2017;19(10):1260-73.

36. Matsuzawa Y, Oshima S, Takahara M, Maeyashiki C, Nemoto Y, Kobayashi M, Nibe Y, Nozaki K, Nagaishi T, Okamoto R, et al. TNFAIP3 promotes survival of CD4 T cells by restricting MTOR and promoting autophagy. Autophagy. 2015;11(7):1052-62.

37. Bareau B, Rey J, Hamidou M, Donadieu J, Morcet J, Reman O, Schleinitz $\mathrm{N}$, Tournilhac O, Roussel M, Fest T, et al. Analysis of a French cohort of patients with large granular lymphocyte leukemia: a report on 229 cases. Haematologica. 2010;95(9):1534-41.

38. Barilà G, Teramo A, Calabretto G, Vicenzetto C, Gasparini VR, Pavan L, Leoncin M, Vedovato S, Frigo AC, Facco M, et al. Stat3 mutations impact on overall survival in large granular lymphocyte leukemia: a single-center experience of 205 patients. Leukemia. 2020;34(4):1116-24.

\section{Publisher's Note}

Springer Nature remains neutral with regard to jurisdictional claims in published maps and institutional affiliations. 